\title{
Time Series Analysis for Assessing and Forecasting of Road Traffic Accidents - Case Studies
}

\author{
THEODOR D. POPESCU \\ National Institute for Research and Development in Informatics Society-Oriented IT Systems and Applications \\ Department \\ 8 - 10 Averescu Avenue, 011455 Bucharest \\ ROMANIA
}

\begin{abstract}
Road traff c accident is one of the main causes of injuries and fatalities worldwide, serious injuries and mortality in road collisions being a public health problem. The paper gives a overview of time series modeling and forecasting with application in road traff $\mathrm{c}$ injuries monitoring. After presenting of the main models and the methodological issues used in Box-Jenkins approach, the paper discusses two case studies, using a multiplicative $S A R I M A$ model and an intervention model, for a time series representing the number of mortal traff c accidents in USA, and the road traff $\mathrm{c}$ accidents with death and serious injuries in the UK, before and after the imposition of the Arabian embargo in November 1973.
\end{abstract}

Key-Words: Time series analysis, Modeling, Forecasting, Intervention analysis, Box-Jenkins approach, Road traf$\mathrm{f} \mathrm{c}$ accidents.

Received: November 7, 2019. Revised: April 30, 2020. Accepted: May 11, 2020. Published: May 26, 2020.

\section{Introduction}

Road traff c accident is one of the main causes of injuries and fatalities worldwide. According to the World Medical Association, (2006), [1], serious injuries and mortality in road collisions are a public health problem with consequences similar to those of major diseases such as cancer and cardiovascular disease. It is estimated that road traff $\mathrm{c}$ accident will become the 6th leading cause of death in the world and take the 3rd place of disability by the year 2020, [2]. Injuries, deaths and disabilities resulting are considered as a major public heath concerns to which inadequate attention has been paid so far, [3], [4].

Road traff $\mathrm{c}$ accidents has a decreasing trend in developed countries, but a higher number of injuries is reported in developing nations, [4], [5], [6], [7]. Injuries due to road traff $\mathrm{c}$ accidents are one of the main health care problems, which are preventable as the experience of many developed countries. The prevention and control of health events implies implementation of appropriate programs, and adoption of laws (e.g. seat belt law among others) in the legislation on the mortalities resulting from car accidents, etc. Trend assessment and forecasting the data can provide useful information to increase the quality of decision in this f eld. There are different statistical methods to forecast mortality and serious injuries resulting from traf$\mathrm{f} \mathrm{c}$ accidents. A such statistical method is time series analysis, whose main purpose is modeling and fore- casting of data provided from road traff $\mathrm{c}$ accidents.

A time series is def ned as a sequence of measurements, usually equally spaced and ordered in time. Statistical methods applied to time series data were originally developed mainly in econometrics, but they are used in many other f elds, such as physics and engineering, environment, medicine, etc. The f rst applications in this feld were in forecasting, the purpose being to produce an accurate forecast of the future data or measurements for an observed time series.

Applications of time series analysis in road traff c mortality and serious injuries are reported in [8], [9], [10], among others.

The paper is organized as follows. In Section 2 is given a general view on the time series models, regression and intervention models, to be used in modeling and forecasting of road traff $\mathrm{c}$ injuries. Section 3 discusses some methodological aspects of time series modeling and forecasting, based on Box-Jenkins methodology, with the emphasis on practical aspects. Section 4 presents a case study using a multiplicative $A R I M A$ model for a time series representing the number of mortal traff c accidents, monthly recorded, in USA in the period 1973-1978, while the Section 5 has as object modeling of a interrupted time series, an example of intervention analysis, using the road traff $\mathrm{c}$ accidents with death and serious injuries in the UK, before and after the imposition of the Arabian embargo in November 1973. 


\section{Time series models}

The statistical approaches adopted in time series modeling and forecasting usually rely on multiplicative $S A R I M A$ (Seasonal Auto Regressive Integrated Moving Average) model. A such model has the following form for the time series $z_{t}$, [11]:

$$
\phi(B) \Phi\left(B^{s}\right) \nabla^{d} \nabla_{s}^{D} z_{t}=\theta(B) \Theta\left(B^{s}\right) a_{t}
$$

where $a_{t}$ is a white noise and

$$
\begin{aligned}
& \phi(B)=1+\phi_{1} B+\phi_{2} B^{2}+\cdots+\phi_{p} B^{p} \\
& \theta(B)=1+\theta_{1} B+\theta_{2} B^{2}+\cdots+\theta_{q} B^{q} \\
& \Phi\left(B^{s}\right)=1+\Phi_{s} B^{s}+\Phi_{2 s} B^{2 s}+\ldots+\Phi_{P s} B^{P s} \\
& \Theta\left(B^{s}\right)=1+\Theta_{s} B^{s}+\Theta_{2 s} B^{2 s}+\ldots+\Theta_{Q s} B^{Q s}
\end{aligned}
$$

with $B$ the time delay operator, $B z_{t}=z_{t-1}, \nabla z_{t}=$ $(1-B)=z_{t}-z_{t-1}$, nonseasonal differentiating operator, and $\nabla_{s} z_{t}=\left(1-B^{s}\right)=z_{t}-z_{t-s}$, seasonal differentiating operator: $d$ is the nonseasonal differentiating order, $D$ is the seasonal differentiating order and $s$ is the seasonal period of the series.

The model is defned as $S A R I M A(p, d, q)(P, D, Q)_{s}$ where $(p, d, q)$ denotes nonseasonal orders, and $(P, D, Q)$ seasonal order of the model. The model is presented in Fig. 1.

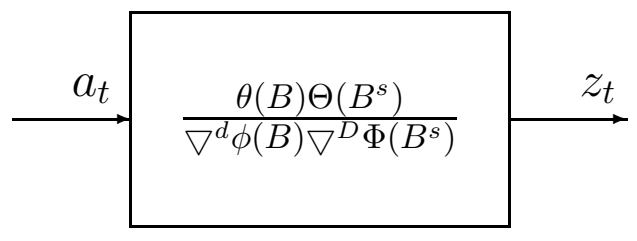

Figure 1: Multiplicative model $S A R I M A$ $(p, d, q)(P, D, Q)_{s}$

The multiplicative form of the model simplif es the stationarity and invertibility conditions checking; these conditions can be separately checked, for seasonal and nonseasonal coeff cients of the model.

Starting from the general model form of the model $S A R I M A$ it can be obtain related models: $A R$ (Auto Regressive), $M A$ (Moving Average), $A R M A$ (Auto Regressive Moving Average) and ARIMA (Auto Regressive Integrated Moving Average), with or without seasonal components. These models are identif ed by the mean of the autocorrelation $(A C F)$ and the partial autocorrelation functions $(P A C F)$.
In some situations, it is known that some external events can affect the variables for which the practitioner intends to forecast the future time series values. Dynamic models, used in this case, include several variables, as input variables, which are intended to take into account in the dynamics model, the mentioned exception events. A special kind of $S A R I M A$ model with input series is called an intervention model or interrupted time series (ITS) model, [12]. In an intervention model, the input series is an indicator variable that contains discrete values that $f$ ag the occurrence of an event affecting the response series. This event is an intervention in or an interruption of the normal evolution of the response time series, which, in the absence of the intervention, is usually assumed to be a pure $S A R I M A$ process. As examples of practical interventions can be mentioned: the effect of different promotions activities on the sales, the effect of strikes on the volume of the products and the price of the products, the effect of medication on the health of the patient, the effect of the exchange of the laws in the legislation on the mortalities resulting from car accidents, etc. In this case, some variables as step function, consisting of "zero" values and "unit" values, before and after application respectively change policy, medication, or exchange of laws are included in the model, as an external variable.

A such intervention model can be represented like a transfer function ( $T F$ ) model (see Fig. 2), where $z_{t}$ is the value of the endogenous variable at time $t, \mathbf{u}_{t}=$ $\left[u_{1 t}, \ldots, u_{r t}\right]^{T}$ is the vector of exogenous variables, and $a_{t}$ is a white noise error.

$$
\begin{aligned}
& \Omega_{i}(B)=\omega_{i 0}+\omega_{i 1} B+\omega_{i 2} B^{2}+\cdots+\omega_{i n_{i}} B^{n_{i}} ; i= \\
& 1,2, \ldots, r \\
& \Delta_{i}(B)=1+\delta_{i 1} B+\theta_{i 2} B^{2}+\cdots+\delta_{i n \delta_{i}} B^{n \delta_{i}} ; i= \\
& 1,2, \ldots, r
\end{aligned}
$$

$\phi(B), \theta(B), \Phi\left(B^{s}\right)$ and $\Theta\left(B^{s}\right)$ have been described above.

\section{Methodological Aspects}

The time series model construction usually include the following stages, [11]:

- Identif cation (specif cation) of the time series model using some data analysis tools (different graphical representations, autocorrelation functions $(A C F)$ and partial autocorrelation functions $(P A C F))$ in order to determine the types of transformations to obtain stationarity and to estimate the degree of differentiation needed to 


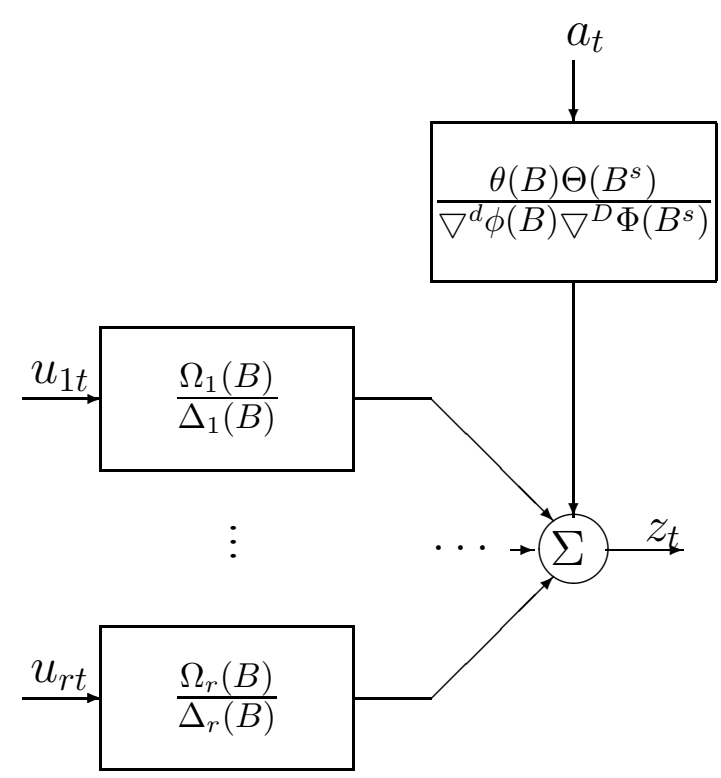

Figure 2: Transfer function $(T F)$ model

induce stationarity in data, as well as the polynomial degrees of autoregressive and moving average operators in the model.

- Model parameter estimation of the time series implies the use of eff cient methods (such as maximum likelihood, among others) for parameter estimation, standard errors and their correlations, dispersion of residuals, etc.

- Model evaluation (validation) aims to establish the model suitability, or to make some simplif cations in structure and parameter estimates. Key elements for model validation refers to residuals which can not be justif ed, these being any residuals of abnormal value that can not be explained by the action of known external factors or other variables; also the correlations and partial correlations of the residuals prove useful tools in model evaluation.

More explanations of the process, e.g. [13], often add a preliminary stage of data preparation and a f nal stage of model application, or forecasting.

Visual analysis of series data allows a frst image on the series' non-stationarity and on the presence of a seasonal pattern in the data. The $f$ nal decision on the inclusion of seasonal elements in the time series model will be taken after the autocorrelation function $(A C F)$ and partial autocorrelation function $(P A C F)$ analysis, as well as after the estimation results analysis; the visual analysis of the data can provide useful additional information.
Signif cant changes in the mean value of the series data require non seasonal differentiation of the f rst order, while the varying of the rate for average value imposes the nonseasonal differentiation of the second order of the series. Strong seasonal variations usually require, not more than the seasonal differentiation of the frst order of the series'data. Autocorrelation function of the series offers information on the nonseasonal and seasonal degrees to be used to obtain the stationarity of the data.

An $A R M A$ stationary process is characterized by theoretical autocorrelation and partial autocorrelation functions tending to zero. The autocorrelation function tends to zero after the frst $q-p$ values of the delay, following the evolution of a exponential function or of a damped sinusoidal function, and the partial autocorrelation function is canceled after the frst $p-q$ values of the delay, [14].

An $A R$ or $M A$ seasonal process is characterized by similar autocorrelation and partial autocorrelation functions, corresponding to nonseasonal processes, but the coeff cients of autocorrelation and partial autocorrelation functions, signif cant for the seasonal process, appear at multiple seasonal delay values.

At the stage of model identif cation a special attention will be given to nonseasonal autocorrelation coeff cients with absolute values of the associated $t$ statistic test exceeding the value 1.6, [14]. Model parameters, associated to these coeff cients prove to be signif cant from the statistical point of view, in the estimation stage.

In the identifcation and validation-diagnosis stages, the attention will be focused on the coeff cients of seasonal autocorrelations with the absolute values of the $t$ statistic test associated which overcome 1.25 value. The seasonal parameters estimates $A R$ or $M A$ , associated to these coeff cients, will appear more signif cant in the estimation stage. If the residual autocorrelation function has zeros values, from statistical point of view, to seasonal delays: $s, 2 s, \ldots$, and to the delays of the form $0.5 s, 1.5 s$, and in the vicinity of seasonal delays: $s+1, s-1,2 s+1,2 s-1, \ldots$, the same warning level will be used: 1.25 . More information on the methodology used in this case can be f nd in [14] and [15].

In the estimation stage, the use of the initial estimates of the model parameters of the value of 0.1 leads to good results in most cases; better initial estimates for model parameters can be obtained based on the autocorrelation and partial autocorrelation functions, used to determine the structure of the model. In this stage as model parameters will be retain those for which $|t| \geq 2$, [14]. The criteria Akaike Information Criterion (AIC), Bayesian information criterion (BIC) or Schwarz information criterion (also SIC, 
SBC, SBIC), [16], Adjusted Root Mean Square Error (ARMSE) and Absolute Mean Percent Error (AMPE), [14], offer information on the parameter estimation quality.

Forecasting is what the whole procedure is designed to accomplish. Once the model has been selected, estimated and checked, it is usually a straight forward task to compute forecasts. The forecasting problem can be solved, in the most direct way, using the multiplicative ARIMA model of the form (1). The description of the model by an inf nitely weighted sum of current values and the earlier noise is proving useful, in particular, to estimate the variance of forecasting values, as well as to determine their conf dence intervals. Standards and practices for time series forecasting are given in [17].

Different forecasting applications, for technical and non-technical systems, using other models, are reported in literature, e.g. [18], [19], [20], among others.

\section{Road Traffic Accidents with Death in the USA}

The analyzed time series represents the number of mortal traff $\mathrm{c}$ accidents, monthly recorded, in USA in the period 1973-1978, [15]. The time series contains 72 values and is represented in Fig. 2.

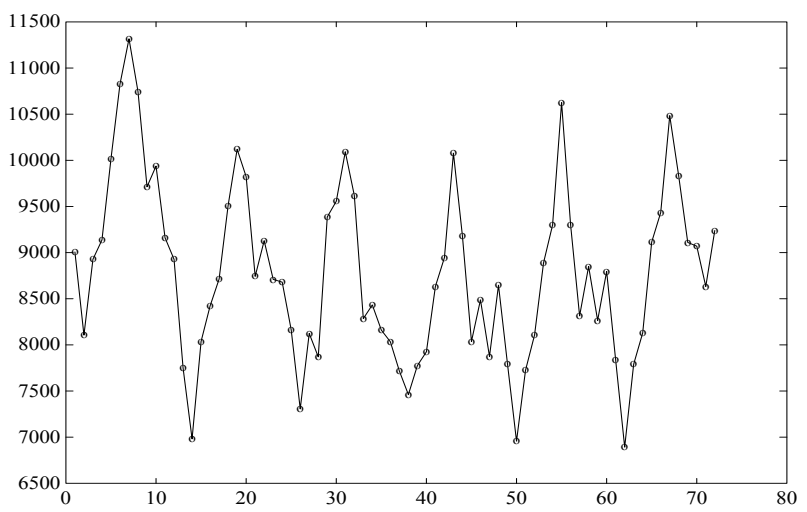

Figure 3: Number of mortal traff c accidents, monthly recorded, in USA: 1973-1978.

The series presents a strong seasonal pattern, with maximum and minimum values in each year, in July and in February, respectively. The presence of a trend component in data is not very clear.

\subsection{Model Identification}

The estimated $A C F$ of the original time series is presented in Fig. 3 and points out the non-stationarity in mean value of data, as well as the presence of a seasonal components of period $s=12$.

The series of nonseasonal differences $(1-B) z_{t}$ is given in Fig. 4.

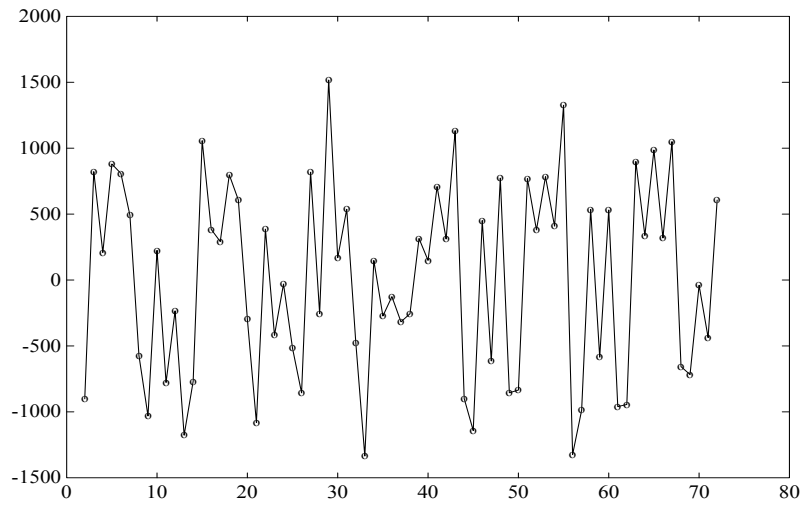

Figure 4: Series of nonseasonal differences $w_{t}=(1-$ $B) z_{t}$.

From $A C F$ analysis, it an be noted the stationarity in mean value of data series and the presence of a seasonal component of period $s=12$, that imposes data seasonal differentiation of $(1-B) z_{t}$ series. The resulted series $w_{t}=\left(1-B^{12}\right)(1-B) z_{t}$ is presented in Fig. 5.

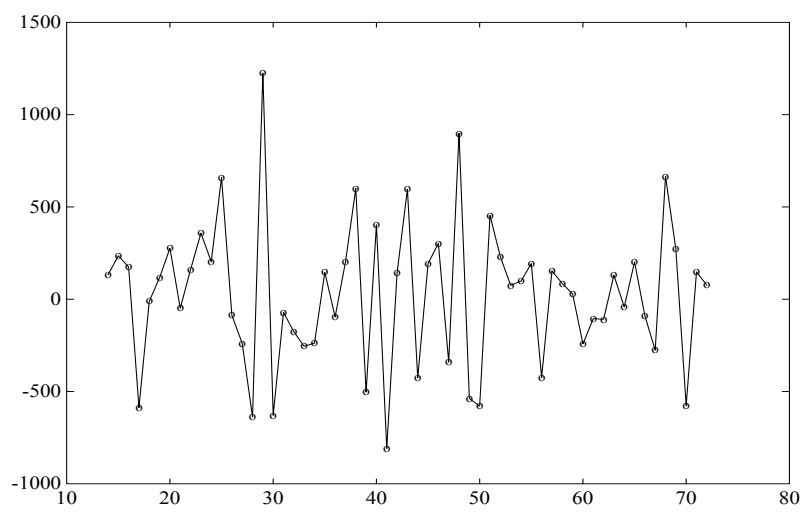

Figure 5: Series $w_{t}=\left(1-B^{12}\right)(1-B) z_{t}$.

It can be noted a data variance decreasing, that justif es the last differentiation operation, but an increasing of the mean value of the series from 3.281 to 28.830 , signif cant reporting to standard deviation value; this suggest to include a constant term, $\theta_{0}$, in the model structure.

The analysis of the frst 11 coeff cients of $A C F$ of $\left\{w_{t}\right\}$ series concludes that only the f rst autocorrelation coeff cient is signif cant different from 0 , from statistical point of view; this suggest to chose for the nonseasonal component of series in multiplicative $A R I M A$ model of a $M A(1)$ factor of the form $\left(1-\theta_{1} B\right)$. This hypothesis is according with slow 
damping of the $A C F$ for reduced values of the delay.

The estimation of the $A C F$ values for the delays 12, 24, 36: $-0.333,-0.099$ and 0.013 , respectively, suggests for seasonal component of the series, in the multiplicative $A R I M A$ model, a $M A(1)_{12}$ factor of the form $\left(1-\Theta_{12} B^{12}\right)$; this hypothesis is according with the slow damping of the $A C F$ for the delays 12, 24 and 36. Consequently, we will adopt, as f rst model form for the original series, one of the form $\operatorname{ARIMA}(0,1,1)(0,1,1)_{12}$ with a constant term $\theta_{0}$ :

$$
(1-B)\left(1-B^{12}\right) z_{t}=\theta_{0}+\left(1-\theta_{1} B\right)\left(1-\Theta_{12} B^{12}\right) a_{t}
$$

\subsection{Model Parameter Estimation}

Model parameter estimation results are given in Fig. 6.

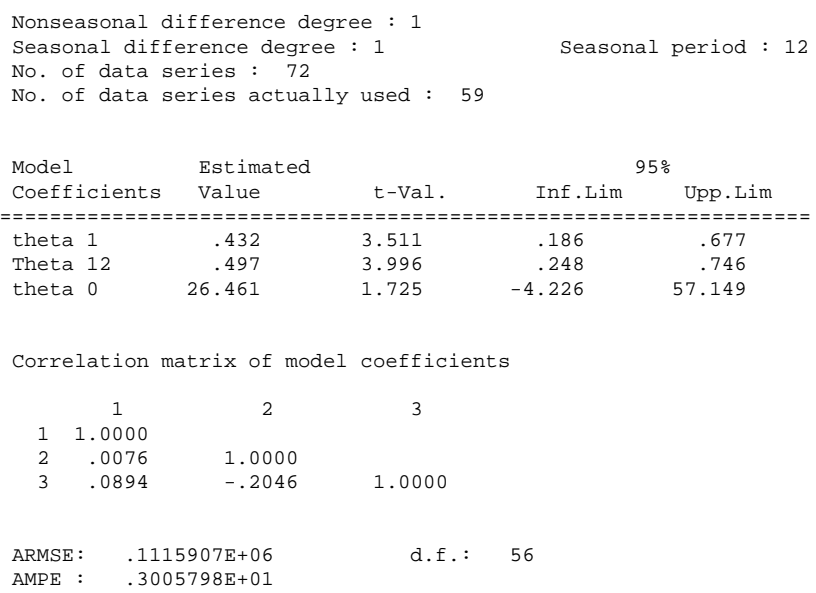

Figure 6: Parameter estimates for model $\operatorname{ARIMA}(0,1,1)(0,1,1)_{12}$ with a constant term $\theta_{0}$.

Model coeff cient estimates are signif cantly different from 0 , from statistical point of view, with absolute values of test statistics $t$, for a signif cance level of $5 \%$, over critical value 2.0 , excepting the coeff cient $\theta_{0}$, slightly below this value (1.725). Also, each of the model coeff cients satisfy the invertibility condition: $\left|\hat{\theta}_{1}\right|<1$ and $\left|\hat{\Theta}_{12}\right|<1$, respectively. The correlation matrix of the model coeff cients shows a very reduced correlation between the coeff cient estimates.

\subsection{Model Validation}

Residuals of model (2) are presented in Fig. 7.

The single value of residual $A C F$ signif cant differently from 0 , from statistical point of view, having

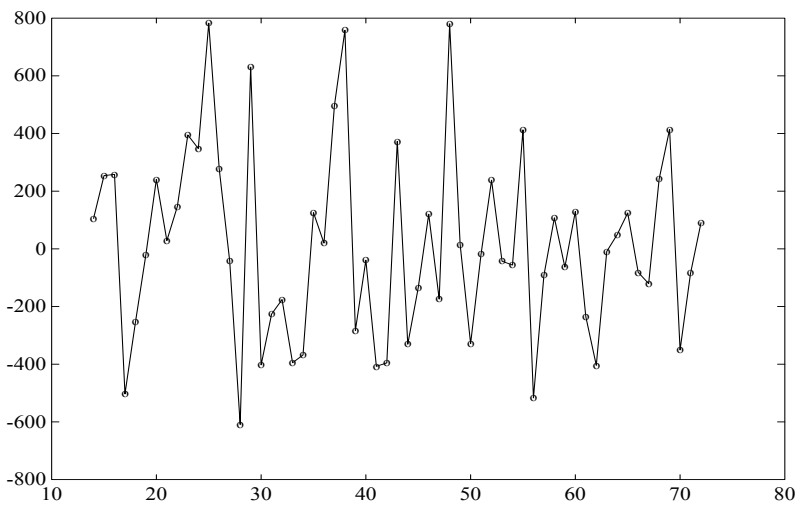

Figure 7: Residuals for model $\operatorname{ARIMA}(0,1,1)(0,1,1)_{12}$ with a constant term $\theta_{0}$.

an absolute value of the statistic test $t$ of 2.038 , at a signif cance level of $5 \%$, is associated with the delay 7. How this delay is of the form $0.5 s+1(s=12)$, it is necessary to reformulate the model of the series. A similar peak appears at the same value of the delay in facp. The statistics value $\chi^{2}$ Ljung-Box, [15], $Q_{L B}$ $=20.377$, is not signif cant for a signif cance level of $5 \%$ and 17 degrees of freedom, and from this point of view the model can be accepted.

\subsection{Model Reformulating, Parameter Esti- mation and Model Validation}

The peak from residual $A C F$, which appears at delay value 7 , suggests inclusion in the model (2) of a term of the form $\theta_{7} B^{7}$, resulting the following model structure for the original data series:

$$
\begin{gathered}
(1-B)\left(1-B^{12}\right) z_{t}=\theta_{0}+\left(1-\theta_{1} B-\theta_{7} B^{7}\right) \times \\
\times\left(1-\Theta_{12} B^{12}\right) a_{t}
\end{gathered}
$$

The estimation results for the model (3) are presented in Fig. 8. It can be noted a reduction of ARMSE and AMPE criteria in comparison with parameter estimation results for model (2).

All model coeff cients have associate absolute values of the statistic test $t$ bigger than 2.0 and are slow correlated. Also, it is satisf ed the necessary condition for invertibility of nonseasonal model component $\left(\hat{\theta}_{1}+\hat{\theta}_{7}<1\right)$, and seasonal component satisf es invertibility condition $\left(\left|\hat{\Theta}_{12}\right|<1\right)$. The model residuals are presented in Fig. 9.

These functions have not values to invalidate the residuals independence, according to Section 3, and consequently the model (3) can be accepted as model for the original data series. 
Non-seasonal difference degree : 1 Seasonal difference degree : 1 No. of data series : 72

No. of data series actually used : 59

\begin{tabular}{lcccc} 
Model & \multicolumn{2}{c}{$\begin{array}{c}\text { Estimated } \\
\text { Coefficients } \\
\text { Value }\end{array}$} & $\begin{array}{l}95 \% \\
\text { t-Val. }\end{array}$ & Inf. Lim. \\
Upp. Lim \\
$====================================================$ \\
theta 1 & .462 & 3.991 & .231 & .694 \\
theta 7 & .244 & 2.033 & .004 & .483 \\
Theta 12 & .536 & 4.285 & .286 & .786 \\
theta 0 & 28.410 & 3.315 & 11.271 & 45.549
\end{tabular}

Correlation matrix of model coefficients

Figure 8: Parameter estimates for the model $\operatorname{ARIMA}(0,1,2)(0,1,1)_{12}$ with a constant term $\theta_{0}$.

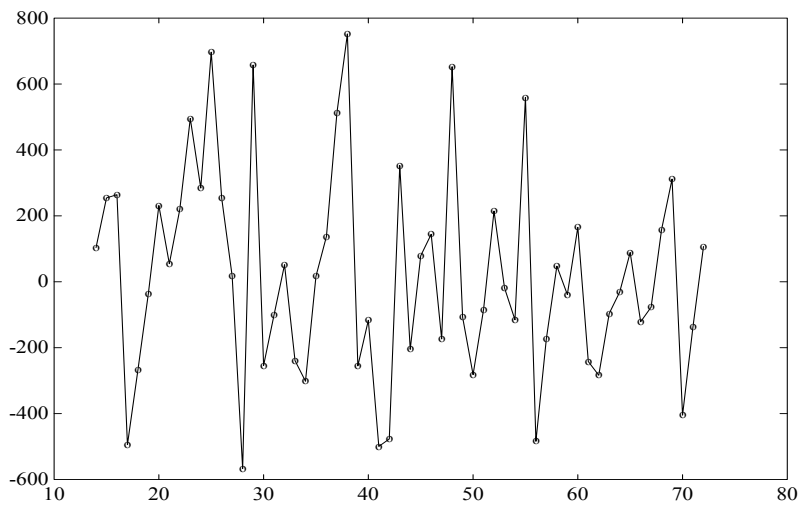

Figure 9: Residual for model $\operatorname{ARIMA}(0,1,2)(0,1,1)_{12}$ with a constant term $\theta_{0}$.

\subsection{Forecasting}

Series forecasting has been performed for the next 6 months using the last determined model. Forecasting results and the real values of the series, are given numerical and graphical in Fig. 10 and Fig. 11, respectively.

\begin{tabular}{lllll}
$\begin{array}{l}\text { Forcasting instant : } 72 \\
\text { Forecasting Forecast. } \\
\text { Value }\end{array}$ & $\begin{array}{l}\text { Real } \\
\text { Value }\end{array}$ & \multicolumn{2}{c}{$\begin{array}{l}\text { Inf. Lim. } \\
\text { horizon }\end{array}==============================================$} \\
1 & $.7798 \mathrm{E}+04$ & $.7812 \mathrm{E}+04$ & $.8444 \mathrm{E}+04$ & $.9075 \mathrm{E}+04$ \\
2 & $.7406 \mathrm{E}+04$ & $.6995 \mathrm{E}+04$ & $.7712 \mathrm{E}+04$ & $.8429 \mathrm{E}+04$ \\
3 & $.8363 \mathrm{E}+04$ & $.7725 \mathrm{E}+04$ & $.8519 \mathrm{E}+04$ & $.9312 \mathrm{E}+04$ \\
4 & $.8460 \mathrm{E}+04$ & $.7916 \mathrm{E}+04$ & $.8779 \mathrm{E}+04$ & $.9642 \mathrm{E}+04$ \\
5 & $.9217 \mathrm{E}+04$ & $.8916 \mathrm{E}+04$ & $.9843 \mathrm{E}+04$ & $.1077 \mathrm{E}+05$ \\
6 & $.9316 \mathrm{E}+04$ & $.9318 \mathrm{E}+04$ & $.1030 \mathrm{E}+05$ & $.1129 \mathrm{E}+05$
\end{tabular}

Figure 10: Forecasting results for a horizon of 6 months using the model $\operatorname{ARIMA}(0,1,2)(0,1,1)_{12}$ with a constant term $\theta_{0}$.

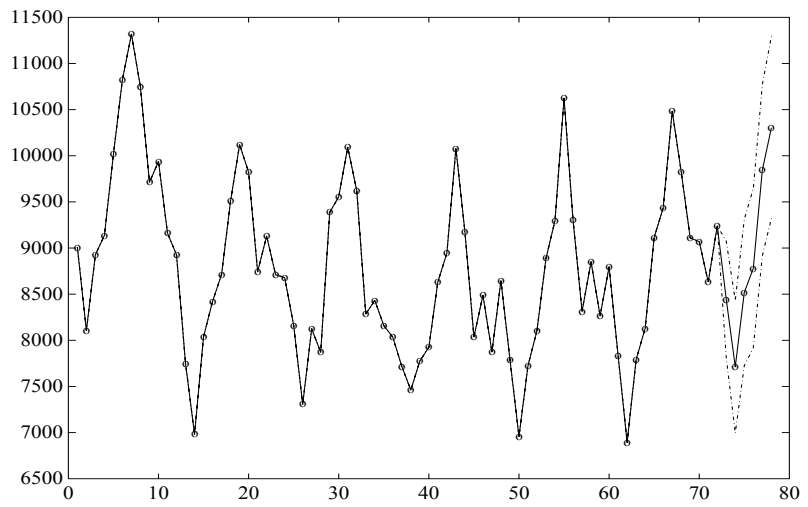

Figure 11: Original series and prediction results for model $A R I M A(0,1,2)(0,1,1)_{12}$ with a constant term $\theta_{0}$

The forecasting is performed on long-term, without taking into account the values of the data series, when they became available.

\section{Road Traffic Accidents with Death and Serious Injuries in the UK}

The time series making the object of the analysis is of the form $z_{t}=\left(1-B^{4}\right) w_{t}-305.2$, where $\left\{w_{t}\right\}$ represents the number of persons in road traff $\mathrm{c}$ accidents in UK with death and serious injuries, registered quarterly, in period 1969-1983. The data $\left\{w_{t}\right\}$ come from [21]; the data also make the object of the analysis in [15], representing a typical case of analysis intervention. The time series is represented in Fig. 12. 


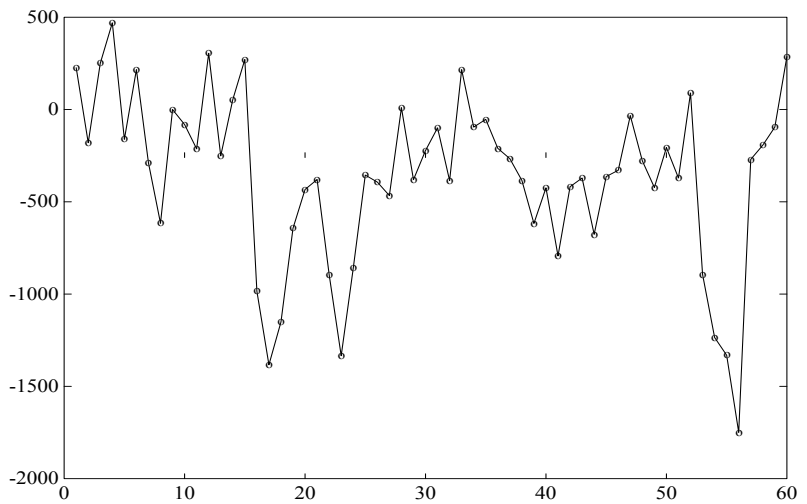

Figure 12: Number of persons in road traff $\mathrm{c}$ accidents in UK with death and serious injuries, registered quarterly: 1969-1983.

\subsection{Model Identification}

From Fig. 12 it can be noted the effect of an intervention on data series $\left\{z_{t}\right\}$, at the 16-th value, corresponding to the imposition of the Arabian embargo in November 1973; the series can be assimilated to an interrupted series (ITS). The data suggest the effect of an intervention of simple change type in level, which can be modeled by a intervention term of the form $\omega \zeta_{t, T}^{(s)}$ with $\zeta_{t, T}^{(s)}$ a step function def ned by $(T=16)$ :

$$
\zeta_{t, T}^{(s)}= \begin{cases}0, & \text { if } 1 \leq t \leq 15 \\ 1, & \text { if } 16 \leq t \leq 60\end{cases}
$$
13.

Intervention variable, $\zeta_{t, T}^{(s)}$, is represented in Fig.

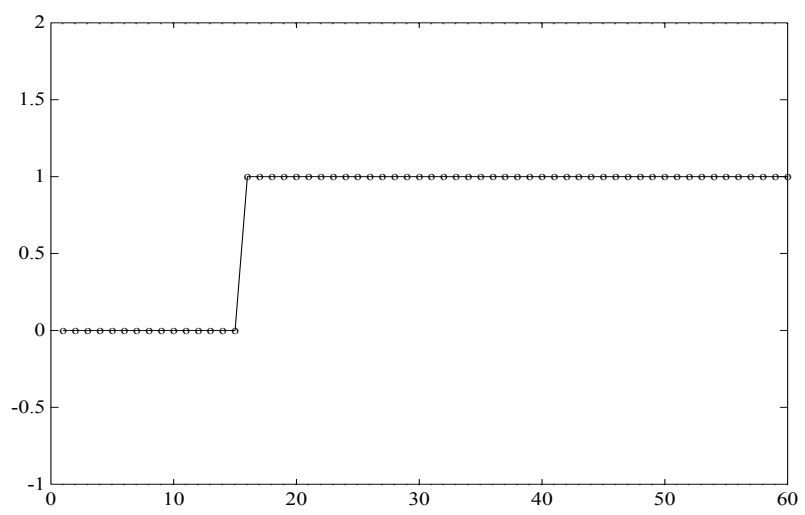

Figure 13: Intervention variable $\zeta_{t, T}^{(s)}$.

\subsection{Parameter Estimation}

For parameter estimation $\omega$ in the model of transfer function type

$$
z_{t}=\omega \zeta_{t, T}^{(s)}+v_{t}
$$

has been used the least squares method $(L S)$, resulting the following model with exogenous variable:

$$
z_{t}=-485.378 \zeta_{t, T}^{(s)}+v_{t}
$$

with $\hat{\sigma}_{v}^{2}=211370$.

The model residuals are given in Fig. 14.

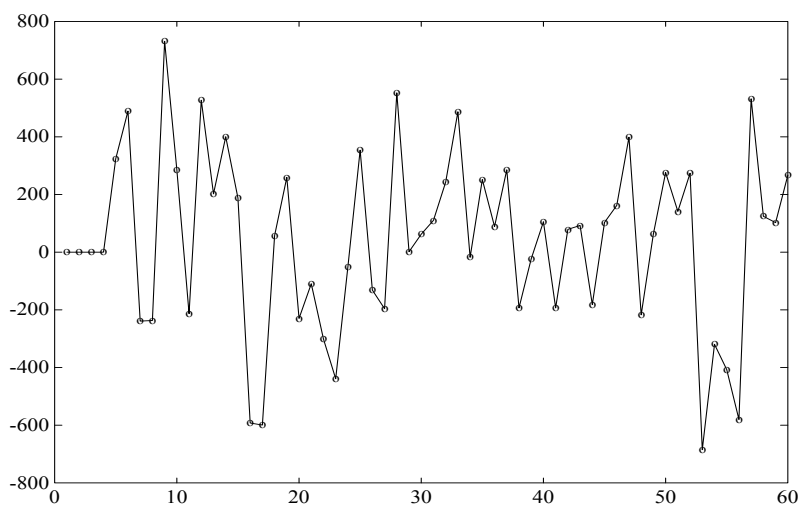

Figure 14: Model residuals.

The residuals of the model (6) have been modeled using the nonlinear least squares methods, resulting the following model for these:

$$
\begin{aligned}
v_{t}= & a_{t}+0.654 a_{t-1}+0.356 a_{t-2}+ \\
& +0.272 a_{t-3}-0.430 a_{t-4}
\end{aligned}
$$

with $\hat{\sigma}_{a}^{2}=93673$, signif cantly reduced in comparison with $\hat{\sigma}_{v}^{2}$.

\subsection{Model Validation}

For the model residuals, $\left\{a_{t}\right\}$, presented in Fig. 14, it can be noted that they do not present extreme values and variations in variance. The residual autocorrelation function was used for the residuals independence hypothesis checking; It can be noted that both, the $t$ statistics analysis, as well as Ljung-Box statistics $\left(Q_{L B}=14.317\right.$ for 15 degrees of freedom $)$ at a level of signif cance of $5 \%$ proved this hypothesis. Consequently, the resulted intervention model can be accepted for the investigated process.

\subsection{Comments}

Knowing the intervention model structure, the parameters of the global model have been directly estimated :

$$
z_{t}=\omega \zeta_{t, T}^{(s)}+v_{t}
$$


where $v_{t}$ has the structure of a $M A(4)$ model. The resulted model was of the form:

$$
\begin{aligned}
z_{t}= & -441.50+a_{t}+0.537 a_{t-1}+0.385 a_{t-2}(9) \\
& +0.277 a_{t-3}-0.423 a_{t-4}
\end{aligned}
$$

Actually tried model parameter estimation for a more general structure which included a term of the form $\phi_{1} z_{t-1}$; this term proved to be insignif cant from a statistical point of view. The variance of the new residuals resulted to be $\hat{\sigma}_{a}^{2}=88141.600$.

From the residual autocorrelation function of resulted model it can be noted, by the $t$ statistics analysis (the values of this statistics are lower than in previous case), as well as by Ljung-Box statistics $\left(Q_{L B}=\right.$ 11.327 for 15 degrees of freedom) at a level of significance of 5\%, that the resulted intervention model can be accepted for the investigated process.

\section{Conclusions}

The time series analysis of road traff $\mathrm{c}$ accidents using multiplicative $A R I M A$ models and the attractive features of the Box-Jenkins approach provide an adequate description to the data in this f eld. The $A R I M A$ processes are a very rich class of possible models and it is usually possible to $\mathrm{f}$ nd a process which provides an adequate description to the data. Monthly pattern was the best time process for forecasting. Also, the intervention analysis proved to be a useful approach to model interrupted time series, in this case, when such time series are generated as the of training drivers to obey traff c laws such as using of the seat belt, some economical constraints, etc. The approach provides a convenient framework which allows an analyst to think about the data, and to f nd an appropriate statistical model which can be used to help answer relevant questions about the data.

\section{Acknowledgments}

The author thanks the Ministry of Research and Innovation for its support under the 2019-2022 Core Program, Project PN 301, RO-SmartAgeing.

\section{References:}

[1] $* * *$ World statement on traff c injury, Adopted by the 42nd World Medical Assembly Rancho Mirage, CA., USA, October 1990 and revised by the WMA General Assembly, Pilanesberg,
South Africa, October 2006, Medical Association (2006).

[2] E. Gomes, R. Araujo, A. Carneiro, C. Dias, F. E. Lecky, A. Costa-Pereira, Mortality distribution in a trauma system: From data to health policy recommendations. Eur J Trauma Emerg Surg. 34 (2008) 561-569.

[3] A. Razzaghi, A. Bahrampour, M. R. Baneshi, F. Zolala, Assessment of trend and seasonality in road accident data: an Iranian case study. Int J Health Policy Manag. 1 (2013) 51-55.

[4] A. BahadorimonfaredA, H. Soori, Y. Mehrabi, A. Delpisheh, A. Esmaili, M. Salehi, et al. Trends of fatal road traff c injuries in Iran (20042011). PLOS ONE. 8 (2013).

[5] M. Esmael, K. Sasaki, K. Nishii, Road traff c accident trend in developing countries-the policy implications. EASTS. 10 (2013) 1978-1990.

[6] E. MendsBrew, J. Dadzie, B. A. Dadson, M. O. Amoamah, Modelling the trend of road traff c accidents in Accra, Mathematical Modelling and Applications, 3 (2018) 1-8.

[7] C. Staton, J. Vissoci, E. Gong, N. Toomey, R. Wafula, J. Abdelgadir, Y. Zhou, C. Liu, F. Pei, B. Zick, C. D. Ratliff, C. Rotich, N. Jadue, L. de Andrade, M. von Isenburg, M. Hocker, Road traff c injury prevention initiatives: A systematic review and metasummary of effectiveness in low and middle income countries, Plos One. (2016) $1-15$.

[8] S. Yousefzadeh-Chabok, F. Ranjbar-Taklimie, R. Malekpouri, A. Razzaghi1, A time series model for assessing the trend and forecasting the road traff c accident mortality, Arch Trauma Res. 5 (2016).

[9] H. Ghaem, M. Hajipour, H. R. Tabatabaee, M. Yadollahi, F. Izanloo, Time series analysis of mortalities resulting from car accidents in injured individuals hospitalized in Shiraz Shahid Rajaee hospital during 2010-2016, Trauma Mon. 23 (2018).

[10] P. Lehohla, Road traff c accident deaths in South Africa, 2001-2006: Evidence from death notif cation, Report No. 03-09-07 (2001-2006) Statistics South Africa (2009).

[11] G. E. P. Box, G. M. Jenkins, Time Series Analysis: Forecasting and Control, 2-nd Edition, Holden Day, San Francisco (1976). 
[12] G. E. P. Box, G. C. Tiao, Intervention Analysis with Applications to Economic and Environmental Problems, Journal of the American Statistical Association, 70 (1975) 70-79.

[13] S. Makridakis, S. C. Wheelwright, R. J. Hyndman (1998) Forecasting: methods and applications , New York: John Wiley \& Sons (1999).

[14] A. Pankratz, Forecasting with Univariate BoxJenkins Models, Wiley, New York (1983).

[15] P. J. Brockwell, R. A. Davis, Introduction to Time Series and Forecasting, Springer-Verlag, New York (1996).

[16] S. Konishi, G. Kitagawa, Information Criteria and Statistical Modeling, Springer (2008).

[17] J. Scott Armstrong, Standards and practices for forecasting, Principles of Forecasting: A Handbook for Researchers and Practitioners, J. Scott Armstrong (ed.), MA: Kluver Academic Publishers (2001) 1-46.

[18] V. Bondarenko, S. Filipova-Petrakieva, I. Taralova, D. Andreev, Forecasting Time Series for Power Consumption Data in Different Buildings Using the Fractional Brownian Motion, International Journal of Circuits, Systems and Signal Processing, 12 (2018) 646-652

[19] L. Di Persio, O. Honchar, Multitask Machine Learning for Financial Forecasting, International Journal of Circuits, Systems and Signal Processing, 12 (2018) 444-451.

[20] J. Liu, J. Bian, H. Li Differential Ant-Stigmergy Algorithm Based Parameters Estimation of Sum of Exponentials Model, International Journal of Circuits, Systems and Signal Processing, 12 (2018) 85-93.

[21] A. Pole, M. West, J. Harrison, Applied Bayesian Forecasting and Time Series Analysis, CRC Press, (1994). 